Links:

Schistosoma mansoni

http://www.ncbi.nlm.nih.gov/ entrez/query.fcgi? $\mathrm{db}=$ genomepr j\&cmd=Retrieve\&dopt=Overvi ew\&list_uids $=12599$

\section{CXCL8}

http://us.expasy.org/uniprot/ P10145

\section{CCL3}

http://us.expasy.org/uniprot/ P10147

\title{
Avoiding attraction
}

The helminth parasite Schistosoma mansoni causes chronic infections in humans, because it can evade host immune defences long-term. Such infections alter the immune response in a manner that prevents the development of various immunemediated diseases, indicating that schistosomes produce immunomodulatory molecules. Now, S. mansoni eggs have been found to secrete a protein that binds certain chemokines and has potent antiinflammatory activity.

Because certain viruses have been shown to produce chemokinebinding proteins (CKBPs) and because infection with $S$. mansoni affects the local recruitment of immune cells, Philip Smith and colleagues examined whether S. mansoni produces CKBPs. Secretions from live eggs (produced by adult worms, which reside in the intestinal blood vessels) were shown to bind the chemokines CXCchemokine ligand 8 (CXCL8; also known as interleukin-8) and CC-chemokine ligand 3 (CCL3). A single protein was found to provide this activity, and the gene that encodes this protein was cloned. This novel CKBP was shown not only to bind specific chemokines but also to prevent these chemokines from interacting with their receptors, thereby inhibiting the migration and activation of leukocytes that express the cognate chemokine receptors (particularly neutrophils, but also macrophages and eosinophils).

This is the first report of a parasite that produces a CKBP. The authors showed that $S$. mansoni CKBP modulates the size and cellular content of the granuloma that forms around the eggs, allowing the eggs to be expelled and thereby maintaining the life cycle of the parasite. In addition, the anti-inflammatory activity of S. mansoni CKBP indicates that it could be used to treat acute inflammation in unrelated situations, and this possibility is supported by data from three in vivo models of acute inflammation.

Davina Dadley-Moore

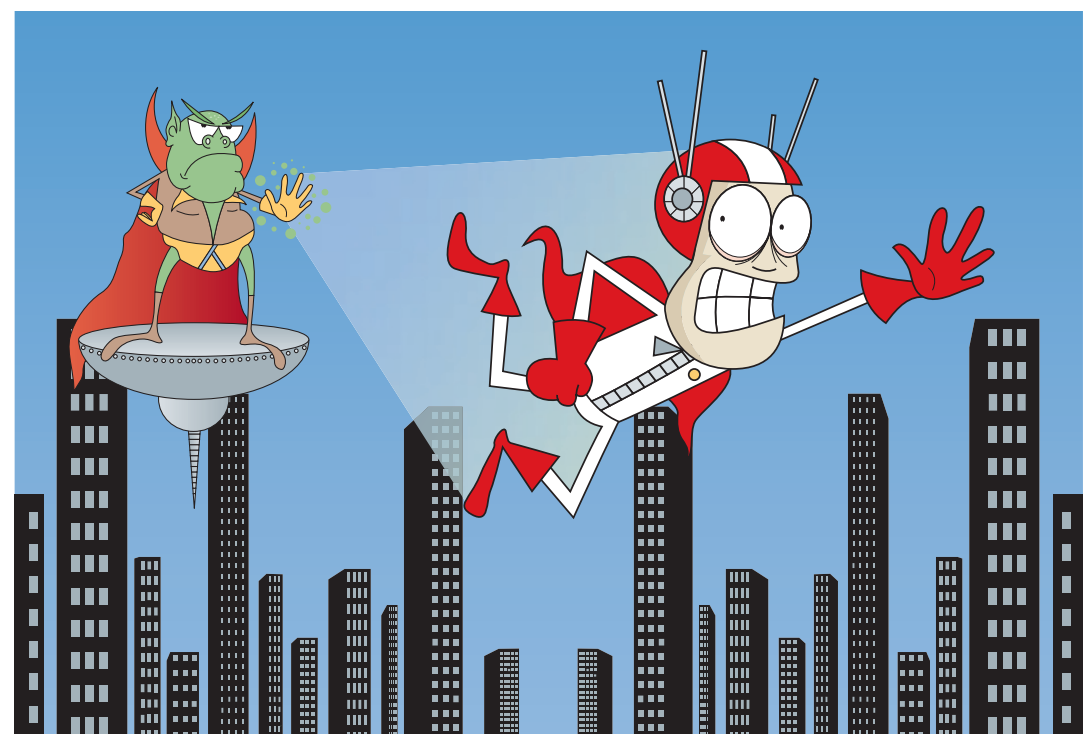

